Frühsommer-Meningoenzephalitis

\section{Rechtzeitig einen Impfschutz aufbauen}

_ Im Frühjahr und Sommer häufen sich die Fälle der Frühsommer-Meningoenzephalitis (FSME). Da nach wie vor keine ursächliche Therapie zur Verfügung steht, raten Experten Erwachsenen und Kindern, die sich vermehrt in der Natur aufhalten, zu einer FSME-Schutzimpfung. Im ersten Quartal des Jahres 2016 wurden in Bayern bereits drei FSME-Fälle registriert. Für den Einzelnen kann eine Infektion schwerste Folgen haben. „Wenn jemand infiziert ist, können wir lediglich Symptome behandeln“, erklärte Dr. Nikolaus Friedrich Frühwein, München.

\section{Jederzeit impfen}

Als Präventionsmaßnahme bietet eine FSME-Schutzimpfung laut Robert-KochInstitut einen „zuverlässigen Schutz gegen die FSME“. Die Grundimmunisierung mit FSME-Immun ${ }^{\circledast}$ erfolgt in drei Teilimpfungen. Nach den ersten beiden
Teilimpfungen besteht ein ausreichender Impfschutz für die aktuelle Zeckensaison. „Die FSME-Schutzimpfung ist eine außerordentlich verträgliche Impfung. Es gibt keine Langzeitnebenwirkungen“, erläuterte Frühwein. „Aus der eigenen Praxis weiß ich, dass eine Tetanusimpfung wesentlich unangenehmer ist als eine FSME-Impfung." Im Gegensatz zu den Angaben der Hersteller, die die Wintermonate als idealen Zeitpunkt zur Immunisierung empfehlen, kann eine Impfung laut Frühwein das ganze Jahr über durchgeführt werden.

\section{Risiko abhängig von der Freizeitgestaltung}

„Je naturexponierter die Freizeitaktivitäten eines Menschen sind, desto höher ist das Risiko einer FSME-Erkrankung. Egal wo Sie leben: Wenn Sie picknicken, wandern oder Fahrrad fahren, sollten Sie sich impfen lassen“, betont Prof. Markus Rose,

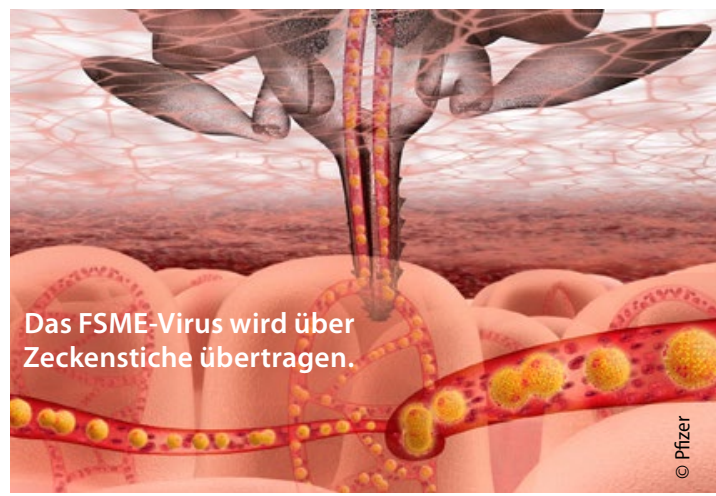

Offenbach am Main. Er rät dazu, auch Kinder impfen zu lassen, denn bei jedem vierten Kind, das an FSME erkrankt, käme es zu schweren Behinderungen, die bis zum Tod führen können. „Diese Infektion, die aus einem gesunden Kind ein schwerstbehindertes Kind macht, sollten wir wirklich verhindern“, appelliert Rose an Eltern und Pädiater.

Julia Rustemeier

- Pressekonferenz „FSME- unterschätztes Infektionsrisiko im eigenen Garten: Relevanz der FSME-Prävention im Praxisalltag"; München, März 2016 (Veranstalter: Pfizer)

\title{
Schweres Asthma
}

\section{Neue Therapien machen orale Steroide zu Mitteln der Reserve}

Bei schwerem Asthma bronchiale erweitern sich die Therapieoptionen: $\mathrm{Zu}$ nehmend werden phänotypspezifische Behandlungen mit Biologika möglich, die ein deutlich besseres Nebenwirkungsprofil aufweisen als orale Steroide (OCS), erklärte Dr. Christian Geßner, Leipzig. Letztere wurden in aktuellen Leilinien zurückgestuft.

Etwa 200.000 Patienten leiden in Deutschland an schwerem Asthma. Es liegt vor, wenn ein Patient orale Steroide über sechs Monate oder hochdosierte inhalative Steroide (ICS) plus weitere Medikamente zur Kontrolle des Asthmas benötigt und darunter eine hohe Krankheitslast bzw. akute Exazerbationen im letzten Jahr aufweist.

OCS sollten nicht langfristig gegeben, mit max. 7,5 mg/d Prednison-Äquivalent dosiert und nur unter Osteoporose-
Monitoring verordnet werden, so Geßner. Das mag schwierig umzusetzen sein, weil es den Patienten unter Steroiden oft gut geht. Die Rechnung kommt aber dann 20 Jahre später: Osteoporose, Hautveränderungen, Diabetes, Katarakt. Geßner empfiehlt, bei Patienten mit schwerem Asthma eine Überweisung zum Spezialisten zu erwägen und ihn im German Asthma Net zu registrieren.

Zur Phänotypisierung ist es notwendig, IgE sowie die Zahl der Bluteosinophilen zu bestimmen. Liegt ein allergisches Asthma vor, sind Allergenkarenz, Immuntherapie und die Therapie mit Omalizumab (Xolair ${ }^{\circledast}$ ) indiziert. „Wir verfügen seit zehn Jahren über gute Alltagserfahrungen. Die Effektivität ist bei geringen Nebenwirkungen hoch. Mehrere Studien zeigen, dass viele Patienten unter IgE-Therapie langfristig weniger oder keine orale Steroide mehr benötigen. “ fasste Geßner zusammen. Er setze Omalizumab bei geeigneten Patienten „spätestens nach der ersten Exazerbation" ein.

Patienten mit nicht-allergischem eosinophilen Asthma sind Kandidaten für den neuen IL-5-Antikörper Mepolizumab. Auch diese Therapie spart orale Steroide ein.

Orale Steroide verbleiben für Patienten, für die Antikörper nicht infrage kommen oder bei denen die Antikörper nicht ausreichend wirksam sind, sowie zur Therapie akuter Exazerbationen.

Dr. Dirk Einecke

- Satellitensymposium „Therapiefortschritt für symptomatische COPD- und Asthmapatienten", 122. Kongress der Deutschen Gesellschaft für Innere Medizin; Mannheim, April 2016 (Veranstalter:Novartis) 